\section{ENFERMEDAD DE MONGE: ¿UN EPÓNIMO EN OLVIDO?}

\section{MONGE'S DISEASE: A FORGOTTEN EPONYM?}

\author{
Alvaro Proaño',a, Eloy F. Ruiz ${ }^{1, a}$
}

Sr. Editor. Hace 89 años, en 1925, el doctor Carlos Monge Medrano publicó en la Academia Peruana de Medicina el primer caso de policitemia en un paciente de Cerro de Pasco, es decir, el primer caso descrito del mal de montaña crónico o también llamado la "Enfermedad de Monge" (1). Este ícono de la medicina peruana no solo destaca en la medicina de altura, también es considerado uno de los padres de la Gastroenterología peruana ${ }^{(2)}$.

Cuando Carlos Monge Medrano empezó a estudiar pacientes con policitemia en Cerro de Pasco él llamó a esta enfermedad "enfermedad de los Andes" o "mal de montaña crónico" (3). En el año 1929, Monge presentó su trabajo en París, lo cual llamó la atención de la comunidad científica, razón por la cual el doctor Louis Henri Vaquez acuñó el término "Enfermedad de Monge" para referirse al mal de montaña crónico ${ }^{(3)}$; epónimo que refleja años de investigación en esta enfermedad.

PubMed es el buscador para la base de datos de Medline más utilizada por los médicos ${ }^{(4)}$. Es por esta razón que sorprende, que luego de 85 años de haberse acuñado el término "Enfermedad de Monge" solo se encuentren diez artículos utilizando la estrategia de búsqueda "Monge`s disease" OR "enfermedad de Monge"

Por otra parte, se sabe que Google Scholar produce el doble de resultados que PubMed ${ }^{(5)}$. Al hacer la misma búsqueda en Google Scholar se encontraron 433 artículos. Esto demuestra claramente que sí existe literatura médica con respecto a este tema. La discrepancia que tiene PubMed no es solo basada en qué revistas busca sino también en cómo las busca. PubMed solo busca el término en el título o en el abstract, pero Google Scholar lo busca en el artículo en sí.

Es por eso que recomendamos que las investigaciones pertinentes al mal de montaña crónico utilicen la frase "Monge's disease" o "enfermedad de Monge" en el título o en el abstract, para que este epónimo pueda ser buscado sin mayores dificultades y no quede en el olvido.

\footnotetext{
Facultad de Medicina, Universidad Peruana Cayetano Heredia. Lima, Perú.

a Estudiante de Medicina.

Recibido: 27-02-14 Aprobado: 12-03-14
}

Citar como: Proaño A, Ruiz EF. Enfermedad de Monge: ¿un epónimo en olvido? [carta]. Rev Peru Med Exp Salud Publica. 2014;31(2):394.
Fuentes de financiamiento: autofinanciado.

Conflictos de interés: los autores declaran no tener conflictos de interés

\section{REFERENCIAS BIBLIOGRÁFICAS}

1. West JB, Schoene RB, Luks AM, Milledge JS. High Altitude Medicine and Physiology. Boca Raton, FL: CRC, 2013.

2. Ramírez Ramos A, Castillo Rosales T. Reseña histórica de la Gastroenterología y endoscopía del aparato digestivo en el Perú: un reconocimiento a los gastroenterólogos que forjaron su desarrollo. Acta Med Per. 2008;25(2):113-22.

3. Rodríguez de Romo AC. Daniel Vergara Lope and Carlos Monge Medrano: two pioneers of high altitude medicine. High Alt Med Biol. 2002 Fall;3(3):299-309.

4. De Leo G, LeRouge C, Ceriani C, Niederman F. Websites most frequently used by physician for gathering medical information. AMIA Annu Symp Proc. 2006;2006:902.

5. Shariff SZ, Bejaimal SA, Sontrop JM, Iansavichus AV, Haynes RB, Weir MA, et al. Retrieving clinical evidence: a comparison of PubMed and Google Scholar for quick clinical searches. J Med Internet Res. 2013 Aug 15;15(8):e164. doi: 10.2196/jmir.2624.

Correspondencia: Alvaro Proaño

Dirección: Av. El Polo 740 Edificio C Oficina 313

Teléfono: 985869820

Correo electrónico: alvaro.proano.f@upch.pe

\section{VIGILANCIA EPIDEMIOLÓGICA, BIOLOGÍA MOLECULAR Y DENGUE 5}

\author{
EPIDEMIOLOGICAL SURVEILLANCE, \\ MOLECULAR BIOLOGY AND DENGUE 5
}
Valeria Ramírez-Jaramillo1,a, Juan Esteban Bedoya-Arias ${ }^{1, a}$, Carlos Enrique Calvache-Benavides ${ }^{1, a}$, Alfonso J. Rodríguez-Morales ${ }^{2,3, \mathrm{~b}}$

Sr. Editor. Hemos leído con interés el artículo de Mamani que discute la importancia de fortalecer la vigilancia molecular del dengue en Perú, en el contexto de la aparición de un nuevo tipo del virus, dengue 5 (DENV-5) ${ }^{(1)}$. Aun cuando concordamos sobre la necesidad existente de orientar esfuerzos que permitan

\footnotetext{
Semillero de Investigación Salud Pública e Infección, Facultad de Ciencias de la Salud, Universidad Tecnológica de Pereira. Risaralda, Colombia.

2 Comité de Zoonosis y Fiebres Hemorrágicas, Asociación Colombiana de Infectología. Bogotá, Colombia.

3 Grupo de Investigación Salud Pública e Infección, Facultad de Ciencias de la Salud, Universidad Tecnológica de Pereira. Risaralda, Colombia.

a Estudiante de Medicina; ${ }^{b}$ médico tropicalista. Recibido: 29-04-14 Aprobado: 07-05-14
}

Citar como: Ramírez-Jaramillo V, Bedoya-Arias JE, Calvache-Benavides CE, Rodríguez-Morales AJ. Vigilancia epidemiológica, biología molecular y dengue 5 [carta]. Rev Peru Med Exp Salud Publica. 2014;31(2):394-5. 
mejorar la identificación molecular de la circulación de serotipos, genotipos y linajes, de forma que se tenga la información oportuna e incorporarla a los sistemas de gestión integrada para el dengue que se viene desarrollando, no solo en Perú sino también en otros países de Latinoamérica, para la prevención y control de esta enfermedad, es importante hacer algunas consideraciones sobre la disponibilidad de dichas herramientas para la epidemiología molecular en la región, así como también de los hallazgos del DENV5 y a quién realmente se atribuye la referencia de dicho descubrimiento, no correctamente planteado por Mamani.

A la fecha (25-04-2014) no existe publicación donde se haya documentado el hallazgo del DENV-5. La referencia citada por Mamani, corresponde a una noticia publicada en la revista Science por un corresponsal (Dennis Normile) ${ }^{(2)}$. No es correcto dejar de citar a la verdadera fuente del hallazgo, Nikos Vasilakis, un virólogo de la University of Texas Medical Branch en Galveston, EUA, el cual en 2013 presentó los hallazgos de dicho nuevo serotipo en la Third International Conference on Dengue and Dengue Haemorrhagic Fever 2013, en Bangkok, Tailandia ${ }^{(3)}$. No fue en un paciente de allá, como indica Mamani, con dengue severo de donde se identificó el serotipo. En realidad fue a partir de muestras de suero recolectadas en 2007 durante una epidemia en Malasia, y que fueron identificadas originalmente como DENV4. Vasilakis secuenció su genoma entero e identificó el nuevo serotipo ${ }^{(2,3)}$. Esto tiene importantes implicaciones epidemiológicas. Más allá de ello, se cree que DENV-5 circularía fundamentalmente en primates no humanos (macacos), por lo cual podría comportarse como una zoonosis.

Es importante conocer la circulación actual de los serotipos en Latinoamérica, más aun porque en muchas ciudades de zonas tropicales, diversas condiciones socioeconómicas y climáticas han permitido una gran carga de enfermedad en la región. Por ello, ciertamente la implantación de métodos de tipificación genética del virus no solo en laboratorios de referencia nacional (lo que ocurre en muchos de nuestros países), sino también en diferentes zonas de los países endémicos, tendrá gran impacto en una potencial detección oportuna del DENV-5 en nuestra región y la importancia en el control de la enfermedad, teniendo en consideración que con la globalización y los viajes es posible su ingreso con un caso importado, que puede conllevar luego a transmisión autóctona. El dengue en viajeros es de reconocida importancia en la actualidad (4).

Finalmente queremos mencionar que estimaciones recientes (2013), basadas en modelos matemáticos, indicarían que la carga de la enfermedad es aún mayor a la que se venía previamente considerando por la Organización Mundial de la Salud, de 50 a 100 millones de casos al año, a 390 millones de casos ${ }^{(5)}$.

Fuente de financiamiento: Universidad Tecnológica de Pereira, Pereira, Risaralda.

Conflictos de interés: los autores declaran no tener conflictos de interés con la publicación de este artículo.

Contribución de autoría: AJRM tuvo la idea de la carta. Todos los autores participaron en la redacción del borrador, en la revisión crítica del manuscrito y aprobaron la versión final a publicar.

\section{REFERENCIAS BIBLIOGRÁFICAS}

1. Mamani E. Nuevo serotipo 5 del virus dengue: necesidad de fortalecer la vigilancia molecular en Perú. Rev Peru Med Exp Salud Publica. 2014;31(1):171-3.

2. Normile D. Tropical medicine. Surprising new dengue virus throws a spanner in disease control efforts. Science. 2013 Oct 25;342(6157):415. doi: 10.1126/science.342.6157.415.

3. Vasilakis $\mathrm{N}$. The daemon in the forest-emergence of a new dengue serotype in South East Asia [Internet]. En: 3rd International Conference on Dengue and Dengue Haemorrhagic Fever 2013. 21-23 October 2013, Bangkok, Thailand [citado el 1 de febrero de 2014]. Disponible en http://www.dengue2013 bangkok.com/uploads/file/Scientific\%20Program\%20 $-17 \% 20$ October\%202013\%20(Final-2).pdf

4. Gallego V, Berberian G, Lloveras S, Verbanaz S, Chaves TSS, Orduna T, et al. The 2014 FIFA World Cup: Communicable Disease Risks and Advice for Visitors to Brazil - a review from the Latin American Society for Travel Medicine (SLAMVI). Travel Med Infect Dis. 2014 May - June;12(3):208-218. doi: 10.1016/j.tmaid.2014.04.004.

5. Bhatt S, Gething PW, Brady OJ, Messina JP, Farlow AW, Moyes $\mathrm{CL}$, et al. The global distribution and burden of dengue. Nature. 2013;496(7446):504-7.

Correspondencia: Alfonso J. Rodríguez-Morales

Dirección: Departamento de Medicina Comunitaria, Piso 3, Facultad de Ciencias de la Salud, Universidad Tecnológica de Pereira, Pereira, Risaralda, Colombia.

Teléfono: 573008847448

Correoelectrónico:arodriguezm@utp.edu.co,ajrodriguezmmd@gmail.com 